\title{
Efectividad de las intervenciones en Funciones Ejecutivas en alumnado con Trastorno del Espectro Autista. Una revisión bibliográfica
}

\author{
Jorge Romero-Martínez - Universitat de València \\ Diana Marín-Suelves - Universitat de València
}

Recepción: 07.02.2021 | Aceptado: 09.02.2021

Correspondencia a través de ORCID: Jorge Romero
0000-0002-0876-8845

0000-0002-5346-8665

Citar: Romero-Martínez, J y Marín-Suelves, D (2020). Efectividad de las intervenciones en Funciones Ejecutivas en alumnado con Trastorno del Espectro Autista. Una revisión bibliográfica. ReiDoCrea, 10(13), $1-15$.

Resumen: El presente trabajo se trata de una revisión bibliográfica a través de la cual se pretende conocer la efectividad de diferentes programas de intervención destinados a potenciar las Funciones Ejecutivas (FE) en el alumnado con Trastorno del Espectro Autista. Se utilizaron varias bases de datos para la selección de los 23 artículos recientes que componen este estudio. La mayoría de los estudios muestran mejoras significativas en las diferentes dimensiones de las FE trabajadas. Aunque hay una cantidad considerable de recursos enfocados a desarrollar las Funciones Ejecutivas, convendría ampliar los estudios y reducir las limitaciones de estos con el fin de que los resultados se puedan universalizar y abarcar a mayor población por la importancia del desarrollo de las funciones ejecutivas para el alumnado con Trastorno del Espectro Autista.

Palabra clave: Necesidades educacionales

Effectiveness of interventions in Executive Functions in students with Autism Spectrum Disorder. A bibliographic review

\begin{abstract}
The present work is a bibliographic review through which it is intended to know the effectiveness of different intervention programs aimed at enhancing the Executive Functions (EF) in students with Autism Spectrum Disorder. Several databases were used for the selection of the 23 recent articles that compose this study. Most of the studies show significant improvements in the different dimensions of the EFs worked on. Although there is a considerable number of resources focused on the development of the Executive Functions, it would be convenient to extend the studies and reduce their limitations so that the results can be universalized and cover a larger population due to the importance of the development of the executive functions for the students with Autism Spectrum Disorder.
\end{abstract}

Keyword: Educational needs

\section{Introducción}

El término "autismo" fue utilizado por primera vez por el psiquiatra y eugenista suizo Euge Bleuer en 1911 en su libro Dementia praecox oder Gruppe der Schizophrenien (Alcantud y Alonso, 2015). Desde entonces este concepto ha sufrido grandes cambios, pasó de ser considerado como una enfermedad mental a principios del siglo XX, a un continuo del Trastorno del Espectro del Autismo, tal y como se le conoce hoy en día (Wing y Gould, 1979)Sin embargo, aunque el término lleva más de un siglo en constante evolución, no fue hasta los años 90 del siglo pasado cuando este comenzó a cobrar un . mayor interés por la comunidad científica. Tan solo es necesario realizar una consulta rápida en bases de datos de publicaciones científicas como en ProQuest Psychology Journals para observar que, si se buscan publicaciones en las que aparezca la palabra "autism", su crecimiento desde esta fecha ha sido exponencial (Alcantud y Alonso, 2015).

Este aumento del interés podría deberse al dramático incremento en la prevalencia del TEA que se ha informado durante los últimos años. Muchas publicaciones afirman la tendencia al alza de este indicador que pasó de ser 1 de cada 2.000/2.500 en los años 
sesenta a 1 de cada 38 o más en las primeras décadas del siglo actual (Matson y Kozlowski, 2011). No obstante, este dato depende de diversas variables como la región geográfica, la edad de la muestra, la fuente a la que se consulte, etc. Así que para tener una visión más amplia sobre la prevalencia es importante conocer otros estudios como el de Baio et al. (2018) que en 2014 obtuvo que 1 de cada 59 niños de Estados Unidos tenía autismo o el de Hill, Zuckerman y Fombonne (2015) que tras analizar un total de 53 estudios en 18 países publicados desde el 2000 hasta el 2014, pudo determinar que la prevalencia actual de TEA ronda el 1/145.

Aunque los datos sobre la prevalencia difieran entre unos estudios y otros, lo que está claro es que número de individuos con autismo ha aumentado recientemente (Chistensen et al., 2016). Este hecho podría explicarse mediante diversas teorías. Autores como Blaxill (2004) apuntan a que el incremento se debe a la mayor incidencia, es decir, que cada vez más personas manifiestan el trastorno. Mientras tanto, otros autores piensan que las causas serían más bien los cambios en los criterios diagnósticos, la mayor divulgación, el mayor conocimiento de la sintomatología por parte de los profesionales y las familias o el desarrollo de mejores métodos de detección (Charman, 2002; Coo et al., 2008; Fombonne, 2003; Wing y Potter, 2002).

En cualquier caso, sea cual sea el motivo de la causa, lo importante es que la cantidad de personas diagnosticadas con TEA es cada vez mayor y, por lo tanto, es necesario que los profesionales estén capacitados para darles una respuesta médica, psicológica y educativa adecuada.

Con el fin de ofrecer dicha respuesta, es importante primero conocer las causas que originan el TEA, lo cual es una tarea complicada. Por ello, a lo largo del tiempo los modelos cognitivos explicativos del trastorno han ido variando. En la actualidad se distingue entre el "autismo idiopático", aquellos casos en los que se desconoce el origen - la causa, y el "autismo sindrómico", cuando la causa es de origen genético o neurológico. Estos últimos tan solo suponen un 15\% aproximadamente de los casos diagnosticados (Artigas, Gabau y Guitart, 2005; Artigas y Diaz, 2013).

Para aquellos casos en los que el origen o la causa es desconocido se han tratado de crear teorías psicológicas. En estas se especula sobre la existencia de alguna estructura neurológica, biológica o un constructo psicológico que sea el responsable de las conductas manifestadas en las personas afectadas. Existen diferentes teorías que han adquirido una gran relevancia como la Teoría de la Mente o la Teoría del déficit en la coherencia central, pero aquella a la que trata de dar una respuesta educativa el presente artículo es a la de la Teoría del déficit en la Función Ejecutiva.

Los primeros trabajos que relacionaron la disfunción en las Funciones Ejecutivas con los síntomas del TEA fueron los de Rumsey (1985), pero fue 6 años más tarde cuando se desarrolló la Teoría del déficit en la FE (Ozonoff, Pennington y Rogers, 1991). Está relación surgió tras observar la analogía entre los problemas de inflexibilidad propios de los individuos que presentan el trastorno y, a su vez, los de las personas con un déficit en las FE.

\section{Objetivos o hipótesis}

A partir de lo anteriormente expuesto, se considera relevante analizar las investigaciones más recientes que se centran en la intervención con alumnado TEA sobre las Funciones Ejecutivas, con el fin de conocer las características de las intervenciones realizadas en los últimos años y así poder establecer unas pautas de actuación educativa en base a la evidencia científica. 


\section{Métodos}

A continuación, se exponen los criterios de inclusión y exclusión a través de los cuales se han seleccionado los artículos presentes en la revisión bibliográfica. En cuanto a los criterios de inclusión fueron los siguientes:

- Artículos publicados en bases de datos de reconocido prestigio, como Scopus, Redalyc y Dialnet.

- Artículos científicos de investigación en los que la muestra este constituida por individuos diagnosticados con TEA.

- Artículos dirigidos a mejorar las FE, ya sea en general o cualquiera de sus dimensiones. Por lo que se ha empleado la combinación de booleans y palabras clave siguiente: autism AND "executive functions" AND intervention AND school OR children.

- Artículos publicados desde 2010 hasta la actualidad (2020), ya que es un rango de búsqueda efectivo para encontrar un número de artículos lo suficientemente amplio a la vez que se evita que estén desactualizados.

- Artículos en los que los participantes tengan edades comprendidas entre los 3 y los 16 años, es decir, las etapas escolares de Educación Infantil, Primaria y Secundaria.

- Artículos redactados en castellano, inglés, italiano o francés.

Respecto a los criterios de exclusión fueron los siguientes:

- Artículos repetidos entre las bases de datos.

- Artículos de acceso restringido o publicados en bases de datos de pago.

- Artículos dirigidos a la edad adulta u otro tipo de trastornos o dificultades de aprendizaje entre los cuales no se contempla el TEA.

- Artículos que no son un programa de intervención educativa o pedagógica. A excepción de aquellos que pueden funcionar como métodos de apoyo a este tipo de intervenciones.

Para realizar el presente trabajo se ha seguido el método propio de las revisiones sistemáticas que se establece en la Declaración PRISMA (Moher, Liberati, Tetzlaff y Altman, 2009). De modo que en la siguiente figura queda relejado el diagrama de flujo surgido de la búsqueda de artículos atendiendo a las directrices de dicho método.

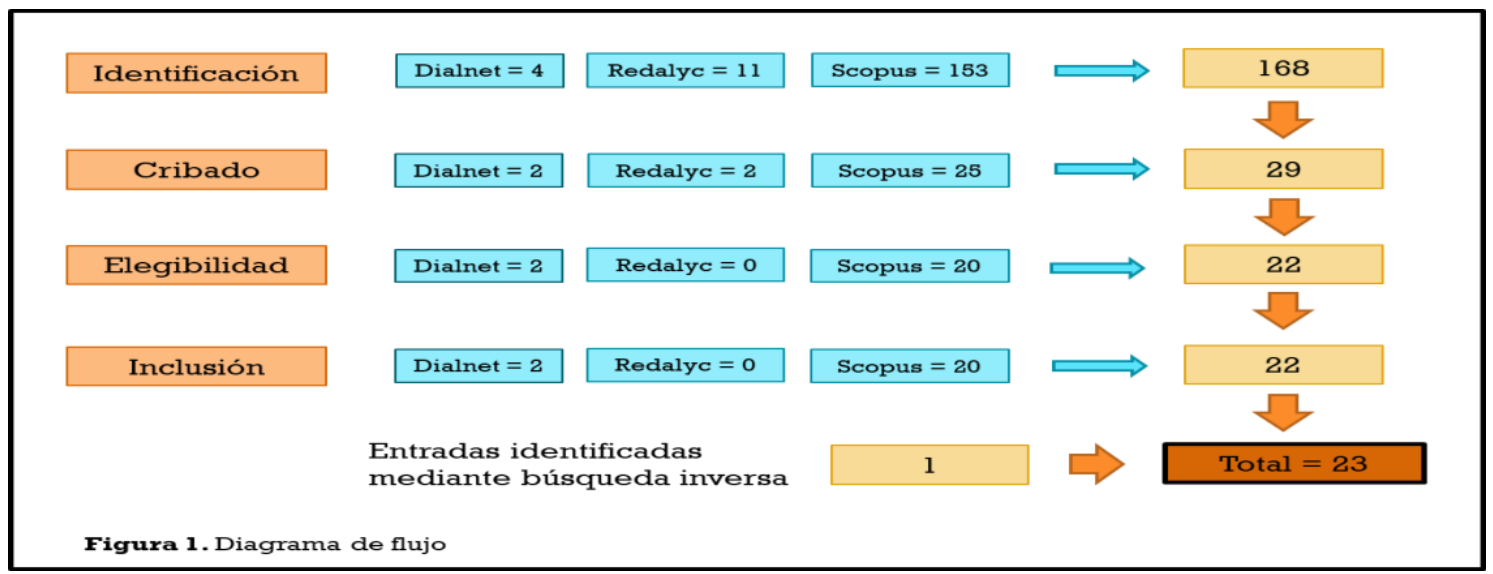

\section{Resultados}

En la Tabla 1 se resumen los rasgos característicos de cada uno de los artículos analizados, como son la autoría, el objetivo principal de la intervención, los participantes, las variables del funcionamiento ejecutivo implicada, los instrumentos de evaluación, la intervención y los resultados obtenidos. 


\begin{tabular}{|c|c|c|c|c|c|c|}
\hline $\begin{array}{l}\text { Autores, año y } \\
\text { origen }\end{array}$ & Objetivo & Muestra & Dimensión variable & Instrumentos & Intervención & Resultados \\
\hline $\begin{array}{l}\text { Baltruschat et al. } \\
\text { (2011) } \\
\text { Estados Unidos }\end{array}$ & $\begin{array}{l}\text { Comprobar si el refuerzo } \\
\text { positivo es capaz de } \\
\text { mejorar la memoria de } \\
\text { trabajo en niños con TEA. }\end{array}$ & $\begin{array}{l}N=3 \text { TEA } \\
S=\hat{O} \\
E=7-11 \text { años }\end{array}$ & $\begin{array}{l}\text { FE: } \\
\text { - Memoria de trabajo }\end{array}$ & $\begin{array}{l}\text { Tarea de dígitos hacia } \\
\text { adelante y hacia atrás } \\
\text { (Hilbert et al., 2015). }\end{array}$ & $\begin{array}{l}\text { Diseño: estudio de caso múltiple. } \\
\text { Procedimiento: se realizaron actividades } \\
\text { para trabajar la memoria de trabajo y se } \\
\text { empleó el refuerzo positivo (objetos o } \\
\text { alimentos) cuando lo hacian bien. } \\
\text { Progresivamente el refuerzo se fue } \\
\text { retirando. } \\
\text { Duración: } 34-65 \text { sesiones. } 2-4 \text { sesiones al } \\
\text { día, 2-3 días a la semana. }\end{array}$ & $\begin{array}{l}\text { Se observaron mejoras en } \\
\text { aquellas tareas que } \\
\text { involucraban a la memoria de } \\
\text { trabajo incluso cuando se } \\
\text { retiró por completo el refuerzo } \\
\text { positivo. }\end{array}$ \\
\hline $\begin{array}{l}\text { Chan et al. } \\
\text { (2011) } \\
\text { China }\end{array}$ & $\begin{array}{l}\text { Analizar el efecto de la } \\
\text { DMBI en las FE de un } \\
\text { individuo con TEA de bajo } \\
\text { funcionamiento. }\end{array}$ & $\begin{array}{l}\mathrm{N}=1 \mathrm{TEA}+\mathrm{DI} \\
\mathrm{S}=\hat{0} \\
\mathrm{E}=9 \text { años }\end{array}$ & $\begin{array}{l}\text { FE: } \\
\text { - Inhibición } \\
\text { - Memoria de trabajo } \\
\text { - Flexibilidad cognitiva }\end{array}$ & $\begin{array}{l}\text { - HKLLT (Chan, 2006). } \\
\text { - CCTT (Williams et al., } \\
\text { 1995). } \\
\text { - BRIEF (Gioia et al., } \\
\text { 2000). }\end{array}$ & $\begin{array}{l}\text { Diseño: estudio de caso único. } \\
\text { Procedimiento: consistió en un cambio en } \\
\text { la dieta, la aplicación de una hierba } \\
\text { intranasal y ejercicios cuerpo-mente. } \\
\text { Duración: } 32 \text { semanas. }\end{array}$ & $\begin{array}{l}\text { El individuo mejoró en las tres } \\
\text { dimensiones de las FE } \\
\text { evaluadas, es decir, la } \\
\text { inhibición, la memoria de } \\
\text { trabajo, y la flexibilidad } \\
\text { cognitiva. }\end{array}$ \\
\hline $\begin{array}{l}\text { Baltruschat et al. } \\
\text { (2012) } \\
\text { Estados Unidos }\end{array}$ & $\begin{array}{l}\text { Comprobar si el refuerzo } \\
\text { positivo es capaz de } \\
\text { mejorar la memoria de } \\
\text { trabajo en niños con TEA. }\end{array}$ & $\begin{array}{l}N=3 \text { TEA } \\
S=\hat{0} \\
E=6-9 \text { años }\end{array}$ & $\begin{array}{l}\text { FE: } \\
\text { - Memoria de trabajo }\end{array}$ & $\begin{array}{l}\text { Tarea de dígitos hacia } \\
\text { adelante y hacia atrás } \\
\text { (Hilbert et al., 2015). }\end{array}$ & $\begin{array}{l}\text { Diseño: estudio de caso múltiple. } \\
\text { Procedimiento: se realizaron actividades } \\
\text { para trabajar la memoria de trabajo y se } \\
\text { empleó el refuerzo positivo (objetos o } \\
\text { alimentos) cuando lo hacian bien. } \\
\text { Progresivamente el refuerzo se fue } \\
\text { retirando. } \\
\text { Duración: } 32-74 \text { sesiones. } 2-4 \text { sesiones al } \\
\text { día, 2-3 días a la semana. }\end{array}$ & $\begin{array}{l}\text { Se observaron mejoras en } \\
\text { aquellas tareas que } \\
\text { involucraban a la memoria de } \\
\text { trabajo incluso cuando se } \\
\text { retiró por completo el refuerzo } \\
\text { positivo. }\end{array}$ \\
\hline $\begin{array}{l}\text { Chan, Sze, Han } \\
\text { y Cheung } \\
\text { (2012). } \\
\text { China }\end{array}$ & $\begin{array}{l}\text { Investigar el efecto de una } \\
\text { modificación dietética } \\
\text { basada en la medicina } \\
\text { Shaolin sobre la mejora de } \\
\text { las FE y los síntomas } \\
\text { conductuales de personas } \\
\text { con TEA. }\end{array}$ & $\begin{array}{l}\mathrm{N}=24 \mathrm{TEA} \\
\mathrm{GE}=12 \\
\mathrm{~S}=100^{\lambda} \text { y } 2 \text { } \\
\mathrm{GC}=12 \\
\mathrm{~S}=100^{\lambda} \text { y } 2 \text { ? } \\
\mathrm{E}=9-14 \text { años }\end{array}$ & $\begin{array}{l}\text { FE: } \\
\text { - Atención } \\
\text { - Flexibilidad mental } \\
\text { - Inhibición } \\
\text { - Planificación }\end{array}$ & $\begin{array}{l}\text { - d2 (Brickenkamp y } \\
\text { Zillmer, 1998) } \\
\text { - CCTT (Williams et al., } \\
\text { 1995). } \\
\text { - ToC (Delis et al., 1997). } \\
\text { - Tarea Go/No Go } \\
\text { (Uzefovsky et al., 2016). } \\
\text { - FPT (Regard, Strauss y } \\
\text { Knap, 1982). }\end{array}$ & $\begin{array}{l}\text { Diseño: estudio de dos grupos con } \\
\text { asignación aleatoria (ensayo controlado } \\
\text { aleatorizado). } \\
\text { Procedimiento: los individuos tan solo } \\
\text { tuvieron que cambiar la dieta. Evitar } \\
\text { alimentos como cebolla, huevo, carne, } \\
\text { pescado, etc. y tratar de consumir otros } \\
\text { como legumbres, fruta, verdura, frutos } \\
\text { secos, etc. } \\
\text { Duración: } 4 \text { semanas. }\end{array}$ & $\begin{array}{l}\text { En el GE se observaron } \\
\text { mejoras significativas en la } \\
\text { planificación, la flexibilidad } \\
\text { mental y la inhibición, sobre } \\
\text { todo, en estas dos últimas. El } \\
\text { efecto sobre la atención fue } \\
\text { menor. }\end{array}$ \\
\hline $\begin{array}{l}\text { Naeeimi, } \\
\text { Hosseini, } \\
\text { Biglarian, Amiri y }\end{array}$ & $\begin{array}{l}\text { Comprobar el efecto de la } \\
\text { estimulación audiovisual en }\end{array}$ & $\begin{array}{l}N=41 \mathrm{TEA} \\
\mathrm{GE}=20 \\
\mathrm{GC}=21\end{array}$ & $\begin{array}{l}\text { FE: } \\
\text { - Planificación } \\
\text { - Adaptación al cambio }\end{array}$ & $\begin{array}{l}\text { BRIEF (Gioia et al., } \\
\text { 2000). }\end{array}$ & $\begin{array}{l}\text { Diseño: estudio de dos grupos con } \\
\text { asignación aleatoria (ensayo controlado } \\
\text { aleatorizado). }\end{array}$ & $\begin{array}{l}\text { El GE, a diferencia del GC, } \\
\text { mostró mejoras en la }\end{array}$ \\
\hline
\end{tabular}




\begin{tabular}{|c|c|c|c|c|c|c|}
\hline $\begin{array}{l}\text { Pishyareh } \\
\text { (2013). } \\
\text { Irán }\end{array}$ & $\begin{array}{l}\text { individuos con TEA de alto } \\
\text { funcionamiento. }\end{array}$ & $E=5-8$ años & - Inhibición & & $\begin{array}{l}\text { Procedimiento: a través de unas gafas y } \\
\text { unos auriculares se estimuló a cada } \\
\text { individuo empleando distintas frecuencias } \\
\text { e intensidades de sonido e iluminación. } \\
\text { Duración: } 18 \text { sesiones de } 35 \text { min. durante } \\
6 \text { semanas. }\end{array}$ & $\begin{array}{l}\text { planificación, la adaptación al } \\
\text { cambio y la inhibición. }\end{array}$ \\
\hline $\begin{array}{l}\text { Tachibana et al. } \\
\text { (2013). } \\
\text { Japón }\end{array}$ & $\begin{array}{l}\text { Mejorar las FE y las } \\
\text { habilidades cognitivas de } \\
\text { niños con TEA mediante la } \\
\text { lectura en voz alta. }\end{array}$ & $\begin{array}{l}\mathrm{N}=11 \mathrm{TEA} \\
\mathrm{GE}=6 \\
\mathrm{~S}=4 \hat{\bigcirc} \text { y } 2 \text { } 2 \\
\mathrm{GC}=5 \\
\mathrm{~S}=4 \hat{\text { y } 1} 1 \text { } \\
\mathrm{E}=8-10 \text { años }\end{array}$ & FE en general & $\begin{array}{l}\text { - WCST (Grant y Berg, } \\
\text { 1948; Heaton, 1981). } \\
\text { - Span-board (Wechsler, } \\
\text { 1981). } \\
\text { - RCPM (Raven, Raven y } \\
\text { Court, 1976). }\end{array}$ & $\begin{array}{l}\text { Diseño: estudio de dos grupos con } \\
\text { asignación aleatoria (ensayo controlado } \\
\text { aleatorizado). } \\
\text { Procedimiento: consistió en leer libros en } \\
\text { voz alta y comprender lo que estaban } \\
\text { leyendo. } \\
\text { Duración: } 25 \text { sesiones de } 30 \text { min. durante } \\
5 \text { semanas. }\end{array}$ & $\begin{array}{l}\text { El GE presentó mejoras } \\
\text { significativas en las FE, lo cual } \\
\text { no ocurrió en el GC. }\end{array}$ \\
\hline $\begin{array}{l}\text { Chan, Sze y Han } \\
\text { (2014). } \\
\text { China }\end{array}$ & $\begin{array}{l}\text { Demostrar que una hierba } \\
\text { intranasal patentada es } \\
\text { capaz de mejorar las FE y } \\
\text { la actividad } \\
\text { neuroelectrofisiológica } \\
\text { asociada a los individuos } \\
\text { con TEA. }\end{array}$ & $\begin{array}{l}N=30 \text { TEA } \\
G E=15 \\
S=13 \overbrace{}^{\lambda} \text { y } 2 q \\
G C=15 \\
S=12 \hat{\text { y }} \text { y } 3 q \\
E=9-14 \text { años }\end{array}$ & $\begin{array}{l}\text { FE: } \\
\text { - Inhibición } \\
\text { - Flexibilidad mental } \\
\text { - Planificación }\end{array}$ & $\begin{array}{l}\text { - d2 (Brickenkamp y } \\
\text { Zillmer, 1998) } \\
\text { - CCTT (Williams et al., } \\
\text { 1995). } \\
\text { - ToC (Delis, Kaplan y } \\
\text { Kramer, 1997). } \\
\text { - BRIEF (Gioia et al., } \\
\text { 2000). } \\
\text { - Tarea Go/No Go } \\
\text { (Uzefovsky et al., 2016). }\end{array}$ & $\begin{array}{l}\text { Diseño: estudio de dos grupos con } \\
\text { asignación aleatoria (ensayo controlado } \\
\text { aleatorizado). } \\
\text { Procedimiento: a cada individuo se le } \\
\text { aplicaron } 10 \mathrm{ml} \text { del extracto de la hierba al } \\
\text { día por la vía nasal. } \\
\text { Duración: } 24 \text { semanas. }\end{array}$ & $\begin{array}{l}\text { El GE, a diferencia del GC, } \\
\text { mostró mejoras en las tres } \\
\text { dimensiones de las FE } \\
\text { analizadas. }\end{array}$ \\
\hline $\begin{array}{l}\text { Hilton et al. } \\
\text { (2014). } \\
\text { Estados Unidos }\end{array}$ & $\begin{array}{l}\text { Mejorar el tiempo de } \\
\text { respuesta, las FE y las } \\
\text { habilidades motoras en } \\
\text { niños con TEA mediante el } \\
\text { entrenamiento Makoto } \\
\text { arena. }\end{array}$ & $\begin{array}{l}N=7 \text { TEA } \\
S=5 \text { त y } 2 \text { ? } \\
E=6-14 \text { años }\end{array}$ & $\begin{array}{l}\text { FE: } \\
\text {-Inhibición } \\
\text {-Adaptación al cambio } \\
\text {-Memoria de trabajo } \\
\text {-Planificación } \\
\text { - Organización }\end{array}$ & $\begin{array}{l}\text { BRIEF (Gioia et al., } \\
\text { 2000). }\end{array}$ & $\begin{array}{l}\text { Diseño: estudio de caso múltiple. } \\
\text { Procedimiento: el entrenamiento consistió } \\
\text { en golpear objetivos iluminados con una } \\
\text { pelota lo más rápido posible e ir } \\
\text { incrementando la velocidad. } \\
\text { Duración: } 30 \text { sesiones de } 2 \text { min. durante } \\
10 \text { semanas. }\end{array}$ & $\begin{array}{l}\text { Se observaron mejoras en } \\
\text { todas las FE trabajadas, en } \\
\text { inhibición, adaptación al } \\
\text { cambio, memoria de trabajo, } \\
\text { planificación y organización. }\end{array}$ \\
\hline $\begin{array}{l}\text { Kenworthy et al. } \\
\text { (2014). } \\
\text { Estados Unidos }\end{array}$ & $\begin{array}{l}\text { Evaluar la efectividad de la } \\
\text { intervención UOT (Cannon, } \\
\text { Kenworthy, Alexander, } \\
\text { Werner y Anthony, 2011) } \\
\text { frente a la intervención SS } \\
\text { para trabajar las FE y las }\end{array}$ & $\begin{array}{l}\mathrm{N}=67 \mathrm{TEA} \\
\text { UOT }=47 \\
\mathrm{~S}=400^{\lambda} \text { y } 7 \text { ? } \\
\mathrm{SS}=20 \\
\mathrm{~S}=180^{\lambda} \text { y } 2 \text { ? } \\
\mathrm{E}=7-11 \text { años }\end{array}$ & $\begin{array}{l}\text { FE: } \\
\text { - Resolución de problemas } \\
\text { - Flexibilidad cognitiva } \\
\text { - Planificación } \\
\text { - Organización }\end{array}$ & $\begin{array}{l}\text { - WASI Block Design } \\
\text { - Challenge Task } \\
\text { - BRIEF (Gioia et al., } \\
\text { 2000). }\end{array}$ & $\begin{array}{l}\text { Diseño: estudio de dos grupos con } \\
\text { asignación aleatoria (ensayo controlado } \\
\text { aleatorizado). } \\
\text { Procedimiento: en ambos grupos se } \\
\text { emplearon técnicas como el role-playing, } \\
\text { el juego y videos. }\end{array}$ & $\begin{array}{l}\text { Aunque ambos grupos } \\
\text { obtuvieron mejoras en las } \\
\text { cuatro dimensiones de las FE } \\
\text { trabajadas, las diferencias } \\
\text { entre el grupo UOT y el SS } \\
\text { fueron significativas a favor del }\end{array}$ \\
\hline
\end{tabular}




\begin{tabular}{|c|c|c|c|c|c|c|}
\hline & $\begin{array}{l}\text { habilidades sociales en } \\
\text { alumnos con TEA. }\end{array}$ & & & & $\begin{array}{l}\text { Duración: } 28 \text { sesiones de 30-40 min. } \\
\text { durante } 24 \text { semanas. }\end{array}$ & $\begin{array}{l}\text { primero. Las mejoras en las } \\
\text { habilidades sociales fueron } \\
\text { significativas y equivalentes en } \\
\text { ambos grupos. }\end{array}$ \\
\hline $\begin{array}{l}\text { Borgi et al. } \\
(2015) . \\
\text { Italia }\end{array}$ & $\begin{array}{l}\text { Examinar si la EAT es } \\
\text { eficaz para mejorar la } \\
\text { conducta adaptativa y las } \\
\text { FE en niños con TEA. }\end{array}$ & $\begin{array}{l}N=28 \text { TEA } \\
G E=15 \\
S=0 \\
G C=13 \\
S=\pi \\
E=6-12 \text { años }\end{array}$ & $\begin{array}{l}\text { FE: } \\
\text { - Planificación } \\
\text { - Resolución de problemas }\end{array}$ & TOL (Shallice, 1982). & $\begin{array}{l}\text { Diseño: estudio de dos grupos con } \\
\text { asignación aleatoria (ensayo controlado } \\
\text { aleatorizado). } \\
\text { Procedimiento: las sesiones se dividieron } \\
\text { en una primera fase de preparación, un } \\
\text { paseo con la mano en el caballo, una fase } \\
\text { de equitación y una fase final de cierre. } \\
\text { Duración: } 25 \text { sesiones de } 60-70 \text { min. } \\
\text { durante } 24 \text { semanas. }\end{array}$ & $\begin{array}{l}\text { Los resultados del GE indican } \\
\text { mejoras en la planificación y la } \\
\text { resolución de problemas. }\end{array}$ \\
\hline $\begin{array}{l}\text { de Vries, Prins, } \\
\text { Schmand y } \\
\text { Geurts (2015). } \\
\text { Países Bajos }\end{array}$ & $\begin{array}{l}\text { Comprobar la efectividad } \\
\text { del entrenamiento } \\
\text { Braingame Brain (Prins et } \\
\text { al., 2013) en el trabajo de } \\
\text { las FE de niños con TEA. }\end{array}$ & $\begin{array}{l}\mathrm{N}=90 \mathrm{TEA} \\
\mathrm{MT}=31 \\
\mathrm{FC}=27 \\
\mathrm{C}=32 \\
\mathrm{E}=8-12 \text { años }\end{array}$ & $\begin{array}{l}\text { FE: } \\
\text { - Flexibilidad cognitiva } \\
\text { - Atención } \\
\text { - Memoria de trabajo } \\
\text { - Inhibición }\end{array}$ & $\begin{array}{l}\text { - Tarea CBTT (Corsi, } \\
\text { 1972). } \\
\text { - Gender-emotion switch- } \\
\text { task (de Vries y Geurts, } \\
\text { 2012). } \\
\text { - N-back task (Smith y } \\
\text { Jonides, 1999) } \\
\text { - Number-switch-task } \\
\text { (Cepeda, Cepeda y } \\
\text { Kramer, 2000) } \\
\text { - Stop-task (Logan, 1994). } \\
\text { - SART (Robertson, } \\
\text { Manly, Andrade, } \\
\text { Baddeley y Yiend, } \\
\text { 1997). } \\
\text { - BRIEF (Gioia et al., } \\
\text { 2000). }\end{array}$ & $\begin{array}{l}\text { Diseño: estudio de tres grupos con } \\
\text { asignación aleatoria (ensayo controlado } \\
\text { aleatorizado) } \\
\text { Procedimiento: los niños se dividieron en } \\
3 \text { grupos y cada uno realizó un tipo de } \\
\text { entrenamiento: memoria de trabajo } \\
\text { adaptativo (MT), flexibilidad cognitiva } \\
\text { adaptativo (FC) y control no adaptativo } \\
\text { (C). } \\
\text { Duración: } 25 \text { sesiones de } 50-150 \text { min. } \\
\text { durante } 6 \text { semanas }\end{array}$ & $\begin{array}{l}\text { En todos los grupos se } \\
\text { observaron resultados } \\
\text { similares. Estos son mejoras } \\
\text { en la memoria de trabajo, la } \\
\text { flexibilidad cognitiva y la } \\
\text { atención, pero no en la } \\
\text { inhibición. }\end{array}$ \\
\hline $\begin{array}{l}\text { Suarez, Mata y } \\
\text { Peralbo (2015). } \\
\text { España }\end{array}$ & $\begin{array}{l}\text { Valorar los efectos de un } \\
\text { programa de intervención } \\
\text { basado en TIC para un } \\
\text { sujeto con TEA que se } \\
\text { centra en la Teoría de la } \\
\text { Mente y las FE. }\end{array}$ & $\begin{array}{l}N=1 \text { TEA } \\
S=\lambda \\
E=10 \text { años }\end{array}$ & $\begin{array}{l}\text { FE: } \\
\text { - Memoria de trabajo } \\
\text { - Flexibilidad mental } \\
\text { - Inhibición } \\
\text { - Atención } \\
\text { - Control de interferencias } \\
\text { - Planificación } \\
\text { - Secuenciación temporal }\end{array}$ & $\begin{array}{l}\text { PEBL: } \\
3 \text { tests: } \\
\text { - Tarea CBTT (Corsi, } \\
\text { 1972). } \\
\text { - Tarea Go/No Go } \\
\text { (Uzefovsky, Allison, } \\
\text { Smith y Baron-Cohen, } \\
\text { 2016). }\end{array}$ & 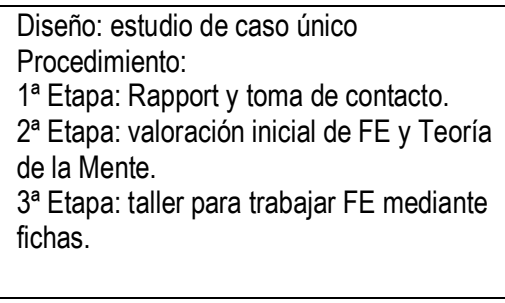 & $\begin{array}{l}\text { Hubo mejoras en la capacidad } \\
\text { de inhibición y mayor } \\
\text { tolerancia a los cambios. } \\
\text { Presentó dificultades para } \\
\text { seguir tiempos marcados y } \\
\text { mayor concentración en las } \\
\text { últimas sesiones. }\end{array}$ \\
\hline
\end{tabular}




\begin{tabular}{|c|c|c|c|c|c|c|}
\hline & & & $\begin{array}{l}\text { - Resolución de problemas } \\
\text { - Autorregulación }\end{array}$ & $\begin{array}{l}\text { - WCST (Grant y Berg, } \\
\text { 1948; Heaton, 1981). }\end{array}$ & $\begin{array}{l}4^{a} \text { Etapa: evaluación final de la aplicación } \\
\text { Aprender Jugando. } \\
\text { Duración: } 13 \text { sesiones de } 90 \text { min. durante } \\
12 \text { semanas. }\end{array}$ & $\begin{array}{l}\text { Realizó todas las tareas } \\
\text { cumpliendo las normas. }\end{array}$ \\
\hline $\begin{array}{l}\text { Bravo y Frontera } \\
(2016) \\
\text { España }\end{array}$ & $\begin{array}{l}\text { Investigar si el } \\
\text { entrenamiento de } \\
\text { diferentes dimensiones } \\
\text { atencionales a través de la } \\
\text { estimulación cognitiva } \\
\text { directa permite la } \\
\text { generalización del } \\
\text { aprendizaje en niños con } \\
\text { TEA. }\end{array}$ & $\begin{array}{l}N=15 \text { TEA } \\
S=14 \hat{\circ} \text { y } 2 q \\
E=7-15 \text { años }\end{array}$ & $\begin{array}{l}\text { FE: } \\
\text { - Atención (mantenida, } \\
\text { selectiva, alternante y } \\
\text { dividida) }\end{array}$ & $\begin{array}{l}\text { - CSAT (Servera y } \\
\text { Llabrés, 2004) } \\
\text { - TMT (Reynolds, 2002) } \\
\text { - AGL (Blanca, } \\
\text { Zalabardo, Rando, } \\
\text { López y Luna, 2005) }\end{array}$ & $\begin{array}{l}\text { Diseño: estudio de caso múltiple. } \\
\text { Procedimiento: consistió en la ejecución } \\
\text { de ejercicios de atención selectiva, } \\
\text { mantenida, dividida y alternante, } \\
\text { regulados por niveles de dificultad. } \\
\text { Duración: } 32 \text { sesiones de } 60 \text { min. durante } \\
24 \text { semanas. }\end{array}$ & $\begin{array}{l}\text { Se obtuvieron mejoras muy } \\
\text { significativas en las cuatro } \\
\text { dimensiones de la atención } \\
\text { evaluadas (mantenida, } \\
\text { selectiva, alternante y } \\
\text { dividida). No existen datos } \\
\text { suficientes para afirmarlo, pero } \\
\text { sí argumentos a favor de la } \\
\text { generalización de este } \\
\text { aprendizaje }\end{array}$ \\
\hline $\begin{array}{l}\text { Didehbani, Allen, } \\
\text { Kandalaft, } \\
\text { Krawczyk y } \\
\text { Chapman } \\
\text { (2016). } \\
\text { Estados Unidos }\end{array}$ & $\begin{array}{l}\text { Investigar el impacto, sobre } \\
\text { todo en el reconocimiento } \\
\text { de emociones, la atribución } \\
\text { social, la atención y las FE, } \\
\text { del VR-SCT en individuos } \\
\text { con TEA de alto } \\
\text { funcionamiento. }\end{array}$ & $\begin{array}{l}\mathrm{N}=30 \\
(17 \text { TEA) } \\
(13 \text { TEA }+ \\
\text { TDAH) } \\
S=26 \oint^{\Uparrow} \text { y } 4 \text { + } \\
E=7-16 \text { años }\end{array}$ & $\begin{array}{l}\text { FE: } \\
\text { - Razonamiento analógico }\end{array}$ & $\begin{array}{l}\text { NEPSY-II Response Set } \\
\text { (Korkman, Kirk y Kemp, } \\
\text { 2007). }\end{array}$ & $\begin{array}{l}\text { Diseño: estudio de caso múltiple. } \\
\text { Procedimiento: en todas las sesiones se } \\
\text { empleó la realidad virtual para crear un } \\
\text { entorno realista y cotidiano en el que } \\
\text { trabajar las habilidades cognitivas y } \\
\text { sociales deseadas. } \\
\text { Duración: } 10 \text { sesiones de } 60 \text { min. durante } \\
5 \text { semanas. }\end{array}$ & $\begin{array}{l}\text { Presentaron mejoras en el } \\
\text { razonamiento analógico } \\
\text { relacionado con las FE. No } \\
\text { hubo diferencias significativas } \\
\text { entre los individuos que } \\
\text { presentaban comorbilidad con } \\
\text { el TDAH y los que no. }\end{array}$ \\
\hline $\begin{array}{l}\text { Acero, Escolano } \\
\text { y Bravo (2017). } \\
\text { España }\end{array}$ & $\begin{array}{l}\text { Mostrar la capacidad de } \\
\text { generalización de } \\
\text { habilidades ejecutivas } \\
\text { aprendidas mediante el } \\
\text { programa de intervención } \\
\text { PIFENA a la vida diaria de } \\
\text { niños con TEA. }\end{array}$ & $\begin{array}{l}\mathrm{N}=7 \\
(5 \mathrm{TEA}) \\
(2 \mathrm{TEA}+\mathrm{DI}) \\
\mathrm{S}=\hat{0} \\
\mathrm{E}=5-12 \text { años }\end{array}$ & $\begin{array}{l}\text { FE: } \\
\text { - Memoria de trabajo } \\
\text { - Planificación } \\
\text { - Inhibición } \\
\text { - Regulación }\end{array}$ & $\begin{array}{l}\text { CHEXI (Thorell y Nyberg, } \\
\text { 2008). }\end{array}$ & $\begin{array}{l}\text { Diseño: estudio de caso múltiple. } \\
\text { Procedimiento: el programa de } \\
\text { estimulación cognitiva directa PIFENA se } \\
\text { implementa individualmente empleando } \\
\text { un diseño de línea de base múltiple. } \\
\text { Posteriormente, se introduce la etapa de } \\
\text { tratamiento. } \\
\text { Duración: } 36 \text { sesiones de } 30 \text { min. durante } \\
12 \text { semanas. }\end{array}$ & $\begin{array}{l}\text { Se mostraron diferencias } \\
\text { estadísticamente significativas } \\
\text { en las cuatro dimensiones de } \\
\text { la FE valorados por el CHEXI } \\
\text { después de la intervención. }\end{array}$ \\
\hline $\begin{array}{l}\text { Hajil et al. } \\
\text { (2017). } \\
\text { Túnez }\end{array}$ & $\begin{array}{l}\text { Analizar si el programa } \\
\text { CRT (Delahunty y Morice, } \\
\text { 1996) sirve para mejorar } \\
\text { las FE, los síntomas } \\
\text { clínicos y los resultados } \\
\text { académicos de personas } \\
\text { con TEA. }\end{array}$ & $\begin{array}{l}\mathrm{N}=16 \text { TEA } \\
\mathrm{E}=7-14 \text { años }\end{array}$ & $\begin{array}{l}\text { FE: } \\
\text { - Flexibilidad cognitiva } \\
\text { - Memoria de trabajo } \\
\text { - Planificación } \\
\text { - Inhibición }\end{array}$ & $\begin{array}{l}\text { - HSCT } \\
\text { - CAAT } \\
\text { - ROCF (Rey, 2941; } \\
\text { Osterrieth, 1944). } \\
\text { - Tests de fluidez y verbal } \\
\text { y semántica. } \\
\text { - Tarea de dígitos hacia } \\
\text { adelante y hacia atrás } \\
\text { (Hilbert et al., 2015). }\end{array}$ & $\begin{array}{l}\text { Diseño: estudio de caso múltiple. } \\
\text { Procedimiento: la intervención se dividió } \\
\text { en } 3 \text { partes de } 8 \text { sesiones cada una. Estas } \\
\text { partes se dedicaron una a trabajar la } \\
\text { flexibilidad cognitiva, otra la memoria de } \\
\text { trabajo y la restante la planificación. Se } \\
\text { emplearon técnicas como el aprendizaje } \\
\text { sin error y el andamiaje. }\end{array}$ & $\begin{array}{l}\text { De las } 4 \text { dimensiones de la FE } \\
\text { analizadas, tan solo se } \\
\text { observaron mejoras } \\
\text { significativas en la memoria de } \\
\text { trabajo. }\end{array}$ \\
\hline
\end{tabular}




\begin{tabular}{|c|c|c|c|c|c|c|}
\hline & & & & & $\begin{array}{l}\text { Duración: } 24 \text { sesiones de } 45 \text { min. durante } \\
26 \text { semanas. }\end{array}$ & \\
\hline $\begin{array}{l}\text { Pan et al. (2017). } \\
\text { China }\end{array}$ & $\begin{array}{l}\text { Examinar los efectos de } \\
\text { una intervención basada en } \\
\text { la actividad física sobre las } \\
\text { habilidades motoras y las } \\
\text { FE de personas con TEA. }\end{array}$ & $\begin{array}{l}N=22 \text { TEA } \\
G E=11 \\
S=0 \\
G C=11 \\
S=0 \\
E=6-12 \text { años }\end{array}$ & FE general & $\begin{array}{l}\text { WCST (Grant y Berg, } \\
\text { 1948; Heaton, 1981). }\end{array}$ & $\begin{array}{l}\text { Diseño: estudio de dos grupos con } \\
\text { asignación aleatoria (ensayo controlado } \\
\text { aleatorizado). } \\
\text { Procedimiento: todas las sesiones se } \\
\text { componian de: calentamiento, habilidades } \\
\text { motoras (algunas enfocadas a trabajar las } \\
\text { FE), juego en grupo y vuelta a la calma } \\
\text { Duración: } 24 \text { sesiones de } 70 \text { min. durante } \\
12 \text { semanas. }\end{array}$ & $\begin{array}{l}\text { El GE a diferencia del GC } \\
\text { mejoró tanto las FE } \\
\text { (rendimiento general, } \\
\text { respuestas perseverantes y } \\
\text { respuestas correctas } \\
\text { consecutivas). }\end{array}$ \\
\hline $\begin{array}{l}\text { Morgan et al. } \\
\text { (2018). } \\
\text { Estados Unidos }\end{array}$ & $\begin{array}{l}\text { Evaluar la eficacia del } \\
\text { modelo SCERTS (Prizant, } \\
\text { Wetherby, Rubin, Laurent y } \\
\text { Rydell, 2006) en } \\
\text { comparación con la } \\
\text { educación habitual de la } \\
\text { escuela a través de ATM } \\
\text { para individuos con TEA. }\end{array}$ & $\begin{array}{l}\mathrm{N}=197 \mathrm{TEA} \\
\mathrm{GE}=118 \\
\mathrm{~S}=93 \partial^{\lambda} \text { y } 25 \text { } \\
\mathrm{GC}=79 \\
\mathrm{~S}=67 \partial^{\lambda} \text { y } 12 \text { } \\
\mathrm{E}=5-8 \text { años }\end{array}$ & FE general & $\begin{array}{l}\text { BRIEF (Gioia et al., } \\
\text { 2000). }\end{array}$ & $\begin{array}{l}\text { Diseño: estudio de dos grupos con } \\
\text { asignación aleatoria (ensayo controlado } \\
\text { aleatorizado). } \\
\text { Procedimiento: se enseño a los maestros } \\
\text { a implementar la CSI en sus aulas y se les } \\
\text { supervisó para que lo implementaran } \\
\text { correctamente. } \\
\text { Duración: } 32 \text { semanas (1 curso escolar). }\end{array}$ & $\begin{array}{l}\text { Los análisis revelaron } \\
\text { resultados significativamente } \\
\text { más altos en el GE que no el } \\
\text { GC en el funcionamiento } \\
\text { ejecutivo, aunque la mejora } \\
\text { fue modesta. }\end{array}$ \\
\hline $\begin{array}{l}\text { Escolano, Acero } \\
\text { y Herrero (2019). } \\
\text { España }\end{array}$ & $\begin{array}{l}\text { Mostrar la utilidad del } \\
\text { enfoque de métodos mixtos } \\
\text { para estudiar las } \\
\text { habilidades de planificación } \\
\text { de individuos con TEA y } \\
\text { evaluar si estas habilidades } \\
\text { mejoran tras la } \\
\text { intervención. }\end{array}$ & $\begin{array}{l}N=8 \\
(4 \text { TEA } \\
\text { Grado 1) } \\
(4 \text { TEA } \\
\text { Grado 2) } \\
S=0 \\
E=5-12 \text { años }\end{array}$ & $\begin{array}{l}\text { FE: } \\
\text { - Habilidades de planificación }\end{array}$ & $\begin{array}{l}\text { Instrumento de } \\
\text { observación creado ad } \\
\text { hoc. }\end{array}$ & $\begin{array}{l}\text { Diseño: estudio de caso múltiple. } \\
\text { Procedimiento: se realizaron } 6 \text { tareas con } \\
8 \text { actividades cada una de dificultad } \\
\text { creciente y relacionadas con acciones } \\
\text { cotidianas. Fueron grabadas y se } \\
\text { evaluaron tanto durante la intervención } \\
\text { como } 1 \text { mes después de finalizarla. } \\
\text { Duración: } 24 \text { sesiones de } 30 \text { min. durante } \\
12 \text { semanas. }\end{array}$ & $\begin{array}{l}\text { Ambos grupos mejoraron sus } \\
\text { habilidades de planificación. El } \\
\text { grupo con TEA Grado } 1 \text { pudo } \\
\text { realizar habilidades de } \\
\text { planificación complejas de } \\
\text { forma autónoma, mientras que } \\
\text { el de Grado } 2 \text { no pudo (con o } \\
\text { sin ayuda). }\end{array}$ \\
\hline $\begin{array}{l}\text { Nakutin y } \\
\text { Gutierrez (2019). } \\
\text { Estados Unidos }\end{array}$ & $\begin{array}{l}\text { Comprobar si la actividad } \\
\text { física beneficia al } \\
\text { rendimiento académico y } \\
\text { las funciones ejecutivas de } \\
\text { estudiantes con TEA. }\end{array}$ & $\begin{array}{l}N=3 \text { TEA } \\
S=2 O^{\top} \text { y } 1 \text { + } \\
E=6-7 \text { años }\end{array}$ & $\begin{array}{l}\text { FE: } \\
\text { - Memoria de trabajo } \\
\text { - Inhibición }\end{array}$ & $\begin{array}{l}\text { - Tarea Go/No Go } \\
\text { (Uzefovsky et al., 2016). } \\
\text { Tareas de dígitos hacia } \\
\text { adelante y hacia atrás } \\
\text { (Hilbert, Nakagawa, Puci, } \\
\text { Zech y Bühner, 2015) }\end{array}$ & $\begin{array}{l}\text { Diseño: estudio de caso múltiple. } \\
\text { Procedimiento: se siguió un diseño de } \\
\text { línea de base múltiple. La actividad física } \\
\text { consistía en } 12 \text { minutos de trote con } \\
\text { intensidad variable seguidos de } 5 \text { minutos } \\
\text { de calma en los que los estudiantes } \\
\text { andaban o estiraban. } \\
\text { Duración: mínimo } 4 \text { sesiones de } 17 \text { min. } \\
\text { durante } 2 \text { semanas. }\end{array}$ & $\begin{array}{l}\text { No presentaron diferencias } \\
\text { significativas en cuanto a las } \\
\text { FE. }\end{array}$ \\
\hline
\end{tabular}




\begin{tabular}{|c|c|c|c|c|c|c|}
\hline $\begin{array}{l}\text { Phung y } \\
\text { Goldberg (2019). } \\
\text { Estados Unidos }\end{array}$ & $\begin{array}{l}\text { Evaluar la efectividad de } \\
\text { las artes marciales mixtas } \\
\text { (MMA) con el fin de mejorar } \\
\text { las FE en individuos con } \\
\text { TEA. }\end{array}$ & $\begin{array}{l}N=34 \text { TEA } \\
G E=14 \\
S=\sigma^{\lambda} \\
G C=20 \\
S=14 \overbrace{}^{\lambda} \text { y } 60 \\
E=8-11 \text { años }\end{array}$ & $\begin{array}{l}\text { FE: } \\
\text { - Inhibición } \\
\text { - Memoria de trabajo } \\
\text { - Flexibilidad cognitiva. }\end{array}$ & $\begin{array}{l}\text { - Hearts \& Flowers test. } \\
\text { - BRIEF (Gioia et al., } \\
\text { 2000). }\end{array}$ & $\begin{array}{l}\text { Diseño: estudio de dos grupos con } \\
\text { asignación aleatoria (ensayo controlado } \\
\text { aleatorizado). } \\
\text { Procedimiento: } \\
1^{\text {a } E t a p a: ~ a p r e n d i e r o n ~ c o m b i n a c i o n e s ~} \\
\text { simples de golpes de los que debían } \\
\text { recordar el orden y el movimiento. } \\
2^{\mathrm{a}} \text { Etapa: se aumentó la complejidad de } \\
\text { los movimientos y se trabajó por parejas. } \\
3^{\mathrm{a}} \text { Etapa: se combinaron todas las } \\
\text { habilidades aprendidas. } \\
\text { Duración: } 26 \text { sesiones de } 45 \text { min. durante } \\
13 \text { semanas. }\end{array}$ & $\begin{array}{l}\text { Según el test Hearts \& } \\
\text { Flowers, los tres dominos de } \\
\text { las FE analizados mejoraron } \\
\text { de forma significativa, aunque } \\
\text { la inhibición conductual en } \\
\text { menor medida. } \\
\text { Los resultados del BRIEF } \\
\text { realizado por los padres } \\
\text { muestran una mejora en la } \\
\text { regulación emocional y } \\
\text { comportamental (inhibición y } \\
\text { autocontrol), pero no en la } \\
\text { regulación cognitiva (memoria } \\
\text { de trabajo y planificación). }\end{array}$ \\
\hline $\begin{array}{l}\text { Tse et al. (2019). } \\
\text { China }\end{array}$ & $\begin{array}{l}\text { Examinar el impacto de la } \\
\text { actividad física en la } \\
\text { calidad del sueño y las } \\
\text { funciones ejecutivas de } \\
\text { sujetos con TEA. }\end{array}$ & $\begin{array}{l}\mathrm{N}=40 \mathrm{TEA} \\
\mathrm{GE}=19 \\
\mathrm{~S}=140^{\lambda} \text { y } 5 \text { q } \\
\mathrm{GC}=21 \\
\mathrm{~S}=180^{\lambda} \text { y } 3 q \\
\mathrm{E}=8-12 \text { años }\end{array}$ & $\begin{array}{l}\text { FE: } \\
\text { - Inhibición } \\
\text { - Memoria de trabajo }\end{array}$ & $\begin{array}{l}\text { - Tarea Go/No Go } \\
\text { (Uzefovsky et al., } \\
\text { 2016). } \\
\text { - Tarea CBTT (Corsi, } \\
\text { 1972). } \\
\text { - Tareas FDS y BDS } \\
\text { (Thorndike, Hagen y } \\
\text { Sattler, 1986). }\end{array}$ & $\begin{array}{l}\text { Diseño: estudio de dos grupos con } \\
\text { asignación aleatoria (ensayo controlado } \\
\text { aleatorizado). } \\
\text { Procedimiento: con el GE se practicaron } \\
\text { diferentes entrenamientos para trabajar } \\
\text { habilidades relacionadas con el } \\
\text { baloncesto mientras que el GC se } \\
\text { mantuvo a la espera. } \\
\text { Duración: } 24 \text { sesiones de } 45 \text { min. durante } \\
12 \text { semanas. }\end{array}$ & $\begin{array}{l}\text { En el GE a diferencia del GC } \\
\text { el control de la inhibición se } \\
\text { vio significativamente } \\
\text { mejorado, pero en la memoria } \\
\text { de trabajo no se apreciaron } \\
\text { diferencias. }\end{array}$ \\
\hline $\begin{array}{l}\text { Parladé et al. } \\
\text { (2020). } \\
\text { Estados Unidos }\end{array}$ & $\begin{array}{l}\text { Comprobar si la } \\
\text { intervención mediante PCIT } \\
\text { es igual de funcional en } \\
\text { individuos con y sin TEA en } \\
\text { el trabajo de los } \\
\text { comportamientos } \\
\text { disrruptivos y la mejora de } \\
\text { las FE. }\end{array}$ & $\begin{array}{l}N=32 \\
G E=16 \\
(T E A+C D) \\
S=0 \\
G C=16 \\
(D T+C D) \\
S=0 \\
E=3-7 \text { años }\end{array}$ & FE general & $\begin{array}{l}\text { - BRIEF (Gioia, Isquith, } \\
\text { Guy y Kenworthy, } \\
\text { 2000). } \\
\text { - BRIEF-P (Gioia, Espy y } \\
\text { Isquith, 2002) }\end{array}$ & $\begin{array}{l}\text { Diseño: estudio de dos grupos con } \\
\text { asignación aleatoria (ensayo controlado } \\
\text { aleatorizado). } \\
\text { Procedimiento: en primer lugar, los padres } \\
\text { recibieron formación en PCIT. } \\
\text { Posteriormente, lo trabajaron en clínicas } \\
\text { bajo la supervisión de terapeutas. Por } \\
\text { último, lo llevaron a cabo desde casa con } \\
\text { revisiones regulares. } \\
\text { Duración: } 10 \text { sesiones de } 60 \text { min. durante } \\
10 \text { semanas. }\end{array}$ & $\begin{array}{l}\text { En ambos grupos se } \\
\text { apreciaron mejoras } \\
\text { estadísticamente significativas } \\
\text { en el FE general según el } \\
\text { informe de los padres. }\end{array}$ \\
\hline
\end{tabular}


AGL: Atención Global-Local; ATM: Autism Training Modules; BDS: Backward Digit Span; BRIEF: Behavior Rating Inventory of Executive Function; BRIEF-P: Behavior Rating Inventory of Executive Function-Preschool Version; CAAT: Colors and Animals Attention Test: CBTT. Corsi Block Tapping Task: CCTT: Children's Color Trails Test: CD. Comportamientos disrruptivos: CHEXI: Childhood Executive Function Inventory: CRT: Cognitive Remediaton Therapy: CSAT: Children Sustained Ate A Sula

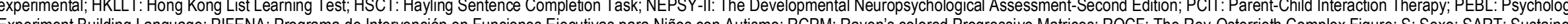
Experiment Building Language; PIFENA: Programa de Intervencion en Funciones Ejecultivas para Niños con Autismo; RCPM. Raven's colored Progressive Matrices; ROCF: The Rey-Osterrieth Complex Figure; S: Sexo; SART: Sustained Making Test; ToC: Tower of California; TOL: Tower of London; UOT: Unstuck an On Target; VR-SCT: Virtual Reality-Social Cognition Training; WASI: Wechsler Abbreviated Scale of Intelligence;WCST: Wisconsin Card Sorting Test 


\section{Discusión}

En este último epígrafe se pretende analizar y comparar la información más relevante extraída de los veintitrés artículos seleccionados para realizar la revisión bibliográfica con el fin de extraer relaciones y conclusiones.

En lo que se refiere al género de la muestra, el número de varones es notablemente superior al de las mujeres. El estudio sociodemográfico desarrollado por la Confederación de Autismo de España (2019), al igual que otros muchos, ha dejado constancia de esta disparidad. De los 2.116 registros recogidos de personas con TEA en España, un 81'43\% de los individuos pertenecen al sexo masculino y un 18 '53\% al femenino, lo cual da lugar a una relación de 1 mujer por cada 4'4 hombres. Estos datos se corresponden con los encontrados en los artículos, aunque en ellos la diferencia es aún más mayor. Sin tener en cuenta los tres trabajos en los que no se detalla el sexo de los participantes (de Vries et al., 2015; Hajril et al. 2017; Naeeimi et al., 2013), hay un 86 ' $16 \%$ de varones (492) frente al 13'84\% de hembras (79), lo cual deja una relación de 1 mujer por cada 6'23 hombres.

Por lo que respecta a la metodología de las intervenciones, algo más de la mitad de los estudios, un 52'17\%, ha optado por el ensayo controlado aleatorizado (Borgi et al., 2015; Chan et al., 2012; Chan et al., 2014; de Vries et al., 2015; Escolano et al., 2019; Kenworthy et al., 2014; Morgan et al., 2018; Naeeimi et al., 2013; Pan et al., 2017; Parladé et al., 2020; Phung y Goldberg, 2019; Tachibana et al., 2013; Tse et al., 2019). Esto denota un alto grado de fiabilidad sobre las evidencias científicas extraídas en cada uno de ellos.

En cuanto a los recursos utilizados para trabajar las Funciones Ejecutivas estos son muy variados. Por lo tanto, los cuatro grandes grupos en los que se pueden dividir para clasificarlos son: aquellas que hacían uso de las TIC, las que se desarrollaban en un aula o en casa mediante actividades relativa o completamente estáticas, aquellas que requerían cierta actividad física o movimiento y las que no se pueden clasificar en ninguno de los anteriores. Los dos más utilizados fueron las actividades en el aula y en casa con materiales simples como, por ejemplo, fichas que se dio en un $43{ }^{\prime} 48 \%$ de los casos (Acero et al., 2017; Baltruschat et al., 2011; Baltruschat et al., 2012; Bravo y Frontera, 2016; Escolano et al., 2019; Hajril et al. 2017; Kenworthy et al., 2014; Morgan et al., 2018; Parladé et al., 2020; Tachibana et al., 2013) y las actividades que requerían cierta actividad física, que se corresponde con el 26'09\% de los casos (Borgi et al., 2015; Hilton et al., 2014; Nakutin y Gutierrez, 2019; Pan et al., 2017; Phung y Goldberg, 2019; Tse et al., 2019).

En relación a las dimensiones de las Funciones Ejecutivas desarrolladas en las intervenciones, las tres que más aparecen en orden decreciente son: la inhibición que aparece en un 52'17\% de los trabajos (Acero et al., 2017; Chan et al., 2011; Chan et al., 2012; Chan et al., 2014; de Vries et al., 2015; Hajril et al. 2017; Hilton et al., 2014; Naeeimi et al., 2013; Nakutin y Gutierrez, 2019; Phung y Goldberg, 2019; Suarez et al., 2015; Tse et al., 2019), la memoria de trabajo en un 47'83\% (Acero et al., 2017; Baltruschat et al., 2011; Baltruschat et al., 2012; Chan et al., 2011; de Vries et al., 2015; Hajril et al. 2017; Hilton et al., 2014; Nakutin y Gutierrez, 2019; Phung y Goldberg, 2019; Suarez et al., 2015; Tse et al., 2019), y la planificación, a veces considerada sinónimo de organización, un 43'48\% (Acero et al., 2017; Borgi et al., 2015; Chan et al., 2012; Chan et al., 2014; Escolano et al., 2019; Hajril et al. 2017; Hilton et al., 2014; Kenworthy et al., 2014; Naeeimi et al., 2013; Suarez et al., 2015). 
Por último, en lo que se refiere a los resultados obtenidos en las diferentes intervenciones, tan solo se tienen en cuenta aquellos relacionados con las Funciones Ejecutivas que es el foco de interés en este trabajo, ya que hay varios artículos que no estaban destinados a trabajar exclusivamente las FE. En primer lugar, aquellos estudios que no obtuvieron ningún tipo de mejora en las FE fueron un 4'35\% (Nakutin y Gutierrez, 2019), los que mostraron mejoras en la FE en general, sin detallar ninguna dimensión, fueron un 17'39\% (Morgan et al., 2018; Pan et al., 2017; Parladé et al., 2020; Tachibana et al., 2013), los que presentaron mejoras en la inhibición fueron un 43'48\% (Acero et al., 2017; Chan et al., 2011; Chan et al., 2012; Chan et al., 2014; Hajril et al. 2017; Hilton et al., 2014; Naeeimi et al., 2013; Phung y Goldberg, 2019; Suarez et al., 2015; Tse et al., 2019), en la planificación un 39'13\% (Acero et al., 2017; Borgi et al., 2015; Chan et al., 2012; Chan et al., 2014; Escolano et al., 2019; Hajril et al. 2017; Hilton et al., 2014; Kenworthy et al., 2014; Naeeimi et al., 2013), en la memoria de trabajo un 34'78\% (Acero et al., 2017; Baltruschat et al., 2011; Baltruschat et al., 2012; Chan et al., 2011; de Vries et al., 2015; Hajril et al. 2017; Hilton et al., 2014; Phung y Goldberg, 2019), en la flexibilidad cognitiva un 34'78\% (Chan et al., 2011; Chan et al., 2012; Chan et al., 2014; de Vries et al., 2015; Hajril et al. 2017; Kenworthy et al., 2014; Phung y Goldberg, 2019; Suarez et al., 2015), en la atención un \% (Bravo y Frontera, 2016; de Vries et al., 2015), en la resolución de problemas un 8'7\% (Borgi et al., 2015; Kenworthy et al., 2014), en la adaptación al cambio un 8'7\% (Hilton et al., 2014; Naeeimi et al., 2013), y en el razonamiento analógico un 4'35\% (Didehbani et al., 2016).

\section{Conclusión}

Uno de los puntos positivos más relevantes extraídos de la revisión es la variabilidad de las intervenciones, tanto en lo que se refiere a las dimensiones de las FE que se potencian como al método o metodología que se sigue. El hecho de que haya cierta variedad en cuanto a la forma de intervenir sirve para poder adecuar el trabajo a cada persona en particular, ya que es fundamental tener en cuenta la gran diversidad existente entre un individuo y otro.

Además, muchos de los estudios también estaban destinados a trabajar otros aspectos como la mejora del sueño (Tse et al., 2019), las habilidades motoras (Borgi et al., 2015; Hilton et al., 2014; Nakutin y Gutierrez, 2019; Pan et al. 2017; Phung y Goldberg, 2019; Tse et al., 2019) o la reducción de los comportamientos disrruptivos (Parladé et al., 2020), algo que es muy útil a la hora de personalizar la intervención, ya que es muy común que las personas con TEA presenten comorbilidad con otros trastornos (Mannion y Leader, 2013).

También cabe destacar los puntos negativos como las limitaciones que tenían muchos de los estudios. Estas son principalmente un tamaño muestral muy reducido; una muestra poco diversa en cuanto a sexo, edad, etnia, sintomatología, etc.; instrumentos de evaluación no testados en la población en la que se han implementado; etc. A pesar de ello, sirven como ruta para futuras investigaciones y, en ocasiones, han incluso creado nuevas líneas de investigación.

Aunque como se acaba de mencionar no existe un tipo de intervención que sea el más adecuado en cualquier circunstancia, puesto que depende del sujeto, aquel que por sus características se podría tomar de referencia es el que se basa en el modelo SCERTS (Social Communication, Emotional Regulation, and Transactional Support) del estudio de Morgan et al. (2018). Las razones principales de esta elección son que incluye una gran cantidad de participantes de diversas etnias, nivel intelectual y grado de afectación sintomática, de modo que es más fácil generalizar los resultados a cualquier individuo; que dura todo un curso escolar; que intervienen tanto los docentes como los padres, así 
que se puede implementar en diversos entornos; que aunque de forma modesta, mejora las FE de manera global; y que también es eficaz para potenciar otros aspectos relevantes como el compromiso activo, la comunicación adaptativa, las habilidades sociales y el comportamiento problemático.

Por último, tan solo queda remarcar la necesidad de, por una parte, ampliar las líneas de investigación y de profundizar en las ya mencionadas tratando de eliminar o reducir las limitaciones, así como, por otra parte, una mayor bibliografía en torno a la mejora de las FE en individuos con TEA. Uno de los motivos por lo que se debe realizar esta ampliación es que cada persona es diferente, cuenta con sus propias particularidades y la mejor forma de encontrar esa metodología que se ajuste a cada uno es aumentar los recursos.

\section{Referencias}

*Acero, M, Escolano, E y Bravo, MA (2017). Generalización del aprendizaje a la vida cotidiana en trastorno del espectro autista a través de un programa de entrenamiento en funciones ejecutivas. Estudios de Psicología, 38(2), 523-536.

Alcantud, F y Alonso, Y (2015). Trastornos del Espectro Autista. En E. M. Moreno (Ed.), Necesidades Educativas Especiales: Una mirada diferente (pp. 1-21). Fedune.

Artigas, J y Diaz, P (2013). Bases biológicas de los trastornos del espectro autista. En F. Alcantud (Coord.), Trastornos del Espectro Autista: Detección, Diagnostico e Intervención Temprana (pp. 35-60). Pirámide.

Artigas, J, Gabau, E y Guitart, M (2005). El autismo sindrómico: I. Aspectos generales. Revista de Neurologia, 40, 143-149.

Baio, J, Wiggins, L, Christensen, DL, Maenner, MJ, Daniels, J, Warren, Z, Kurzius-Spencer, M, Zahorodny, W, Robinson, C, White, T, Durkin, MS, Imm, P, Nikolaou, L, Yeargin-Allsopp, M, Lee, L, Harrington, R, Lopez, M, Fitzgerald, RT, Hewitt, A, ..., \& Dowling, NF (2018). Prevalence of autism spectrum disorder among children aged 8 years-Autism and Developmental Disabilities Monitoring Network, 11 Sites, United States, 2014. Morbidity and Mortality Weekly Report: Surveillance Summaries, 67(6), 1-23.

*Baltruschat, L, Hasselhorn, M, Tarbox, J, Dixon, DR, Najdowski, A, Mullins, RD, \& Gould, E (2011). Addressing working memory in children with autism through behavioral intervention. Research in Autism Spectrum Disorders, 5, 267-276.

*Baltruschat, L, Hasselhorn, M, Tarbox, J, Dixon, DR, Najdowski, A, Mullins, RD, \& Gould, E (2012). The effects of multiple exemplar training on a working memory task involving sequential responding in children with autism. The Psychological Record, 62, 549-562.

Blanca, MJ, Zalabardo, C, Rando, B, López, D y Luna, R (2005). AGL, Atención Global-Local. TEA Ediciones.

Blaxill, M (2004). What's going on? The question of time trends in autism. Public Health Reports, 119(6), 536-551.

*Borgi, M, Loliva, D, Cerino, S, Chiarotti, F, Venerosi, A, Bramini, M, Nonnis, E, Marcelli, M, Vinti, C, De Santis, C, Bisacco, F, Fagerlie, M, Frascarelli, M, \& Cirulli, F (2016). Effectiveness of a standardized equine-assisted therapy program for children with autism spectrum disorder. Journal of autism and developmental disorders, 46(1), 1-9.
*Bravo, MA y Frontera, M (2016). Entrenamiento para la mejora de disfunciones atencionales en niños y adolescentes con Síndrome de Asperger a través de estimulación cognitiva directa. Anales de Psicología, 32(2), 366-373.

Brickenkamp, R, \& Zillmer, E (1998). The d2 test of attention. Hogrefe \& Huber.

Cannon, L, Kenworthy, L, Alexander, KC, Werner, MA, \& Anthony, LG (2011). Unstuck and on target!: An executive function curriculum to improve flexibility for children with autism spectrum disorders. Paul H. Brookes.

Cepeda, NJ, Cepeda, ML, \& Kramer, AF (2000). Task switching and attention deficit hyperactivity disorder. Journal of Abnormal Child Psychology, 28(3), 213-226.

Chan, AS (2006). Hong Kong List Learning Test (2nd Ed.). Department of Psychological and Integrative Neuropsychological Rehabilitation Center.

*Chan, AS, Sze, SL, \& Han, YMY (2014). An intranasal herbal medicine improves executive functions and activates the underlying neural network in children with autism. Research in Autism Spectrum Disorders, 8, 681-691.

*Chan, AS, Sze, SL, Han, YMY, Cheung, MC, Han, YMY, Leung, WWM, \& Shi, D (2011). Dejian mind-body intervention improves the cognitive functions of a child with autism. Evidence-Based Complementary and Alternative Medicine, 2011, 1-7.

${ }^{*}$ Chan, AS, Sze, SL, Han, YMY, \& Cheung, MC (2012). A Chan dietary intervention enhances executive functions and anterior cingulate activity in autism spectrum disorders: A randomized controlled trial. Evidence-Based Complementary and Alternative Medicine, 2012, 1-11.

Charman, T (2002). The prevalence of autism spectrum disorders: Recent evidence and future challenges. European Child and Adolescent Psychiatry, 11(6), 249-256.

Chistensen, DL, Baio, J, Van Naarden, K, Bilder, D, Charles, J, Constantino, JN, Daniels, J, Durkin, MS, Fitzgerald, RT, Kurzius-Spencer, M, Lee, LC, Pettygrove, S, Robinson, C, Schulz, E, Wells, C, Wingate, MS, Zahorodny, W, \& Yeargin, $M$ (2016). Prevalence and Characteristics of Autism Spectrum Disorder Among Children Aged 8 Years. Autism and Developmental Disabilities Monitoring Network, 65(3), 1-23. 
Coo, H, Ouellette, H, Lloyd, J, Kasmara, L, Holden, J, \& Lewis, M (2008). Trends in autism prevalence: diagnostic substitutions revisited. Journal of Autism and developmental Disorders, 38, 1036-1046.

Corsi, P.M (1972). Human memory and the medial temporal region of the brain. Dissertation Abstracts International, 34(2), 819B.

*De Vries, M, Prins, PJM, Schmand, BA, \& Geurts, HM (2015). Working memory and cognitive flexibility-training for children with an autism spectrum disorder: a randomized controlled trial. Journal of Child Psychology and Psychiatry, 56(5), 566-576.

De Vries, M, \& Geurts, HM (2012). Cognitive flexibility in ASD; task switching with emotional faces. Journal of Autism and Developmental Disorders, 42(12), 2558-2568.

Delahunty, A, \& Morice, R (1996). Rehabilitation of frontal/executive impairments in schizophrenia. Australian \& New Zealand Journal of Psychiatry, 30(6), 760-767.

Delis, DC, Kaplan, E, \& Kramer, JH (1997). Delis-Kaplan Executive Function Scale: Standardization edition manual. The Psychological Corporation.

*Didehbani, N, Allen, T, Kandalaft, M, Krawczyk, D, \& Chapman, S (2016). Virtual reality social cognition training for children with high functioning autism. Computers in Human Behavior, 62, 703-711.

*Escolano, E, Acero, M, \& Herrero, L (2019). Improvement of Planning Skills in Children With Autism Spectrum Disorder After an Educational Intervention: A study from a mixed methods approach. Frontiers in Psychology, 10(2824), 1-18.

Fombonne, $E$ (2003). The prevalence of autism. The Journal of the American Medical Association, 289(1), 87-89.

Gioia, GA, Espy, KA, \& Isquith, PK (2002). Behavior Rating Inventory of Executive Function, Preschool Version (BRIEF-P). Psychological Assessment Resources.

Gioia, GA, Isquith, PK, Guy, SC, \& Kenworthy, L (2000). Behavior rating inventory of executive functioning: Manual. Psychological Assessment Resources.

Grant, DA, \& Berg, EAA (1948). A behavioral analysis of degree of reinforcement and ease of shifting to new responses in a Weigel-type card-sorting problem. Journal of Experimental Psychology, 38(4), 404-411.

*Hajri, A., Abbes, Z., Yahia, H. B., Boudali, M., Hamza, M., Bouden, A., Mrabet, A., y Amado, I. (2017). Place of cognitive remedition thearpy in the management of autism spectrum disorder. International Journal of Psychosocial Rehabilitation, 21(1), $55-66$

Heaton, RK (1981). Wisconsin Card Sorting Test Manuel. Psychological Assessment Resource.

Hilbert, S, Nakagawa, TT, Puci, P, Zech, A, \& Bühner, M (2015). The digit span backwards task: Verbal and visual cognitive strategies in working memory assessment. European Journal of Psychological Assessment, 31(3), 174-180.
Hill, AP, Zuckerman, K, \& Fombonne, E (2015). Epidemiology of Autism Spectrum Disorders. In M Robinson (Ed.), Translational Approaches to Autism Spectrum Disorder (pp. 12-38). New York: Springer.

*Hilton, CL, Cumpata, K, Klohr, C, Gaetke, S, Artner, A, Johnson, $\mathrm{H}$, \& Dobbs, S (2014). Effects of exergaming on executive function and motor skills in children with autism spectrum disorder: a pilot study. American Journal of Occupational Therapy, 68, 57-65.

*Kenworthy, L, Anthony, LG, Naiman, DQ, Cannon, L, Wills, MC, Luong-Tran, C, Werner, MA, Alexander, KC, Strang, J, Bal, E, Sokoloff, JL, \& Wallace, GL (2014). Randomized controlled effectiveness trial of executive function intervention for children on the autism spectrum. Journal of Child Psychology Psychiatry, 55(4), 374-383.

Korkman, M, Kirk, U, \& Kemp, S (2007). NEPSY-second edition (NEPSY-II). Harcourt Assessment.

Logan, GD (1994). On the ability to inhibit thought and action: A users' guide to the stop signal paradigm. In D Dagenbach, \& TH Carr (Ed.), Inhibitory processes in attention, memory and language. (pp. 189-239). Academic Press.

Mannion, A, \& Leader, G (2013). Comorbidity in autism spectrum disorder: a literature review. Research in Autism Spectrum Disorders, 7(12), 1595-1616.

Matson, J, \& Kozlowski, A (2011). The increasing prevalence of autism spectrum disorders. Research in Autism Spectrum Disorders 5(1), 418-425.

Moher, D, Liberati, A, Tetzlaff, J, \& Altman, DG (2009). Preferred reporting items for systematic reviews and meta-analyses: the PRISMA statement. Annals of Internal Medicine, 151(4), 264269.

*Morgan, L, Hooker, JL, Sparapani, N, Reinhardt, VP, Schatschneider, C, \& Wetherby, AM (2018). Cluster randomized trial of the classroom SCERTS intervention for elementary students with autism spectrum disorder. Journal of Consulting and Clinical Psychology, 86(7), 631-644.

*Naeeimi, M, Hosseini, SA, Biglarian, A, Amiri, N, \& Pishyareh, E (2013). Effectiveness of audiovisual stimulation on executive function in children with high-functioning autism. Iranian Rehabilitation Journal, 11, 34-39.

*Nakutin, SN, \& Gutierrz, G (2019). Effect of physical activity on academic engagement and executive functioning in children with ASD. School Psychology Review, 48(2), 177-184.

Osterrieth, PA (1944). Le test de copie d'une figure complex: Contribution à l'étude de la perception et de la memoir. Archives de Psychologie, 30, 286-356.

Ozonoff, S, Pennington, B, \& Rogers, S (1991). Executive function deficits in high-functioning autistic individuals: relationship to theory of mind. Journal of Child Psychology and Psychiatry, 37(7), 1081-1105.

*Pan, CY, Chu, CH, Tsai, CL, Sung, MC, Huang, CY, \& Ma, WY (2017). The impacts of physical activity intervention on physical and cognitive outcomes in children with autism spectrum disorder. Autism, 21(2), 190-202. 
*Parladé, MV, Weinstein, A, Garcia D, Rowley, AM, Ginn, NC, \& Jent, JF (2020). Parent-child Interaction therapy for children with autism spectrum disorder and a matched case-control sample. Autism, 24(1), 160-177.

*Phung, JN, \& Goldberg, WA (2019). Promoting executive functioning in children with autism spectrum disorder through mixed martial arts training. Journal of Autism and Developmental Disorders, 49, 3669-3684.

Prins, PJM, Brink, ET, Dovis, S, Ponsioen, A, Geurts, HM, de Vries, M, \& van der Oord, S (2013). "Braingame brian": Toward an executive function training program with game elements for children with ADHD and cognitive control problems. Games for Health Journal, 2(1), 44-49.

Prizant, BM, Wetherby, AM, Rubin, E, Laurent, AC, \& Rydell, JP (2006). The SCERTS Model: Volume I Assessment; Volume II program planning and intervention. Brookes Publishing.

Raven JC, Raven JE, \& Court, JH (1976). Coloured progressive matrices: sets $A, A B, B$. Oxford Psychol Press.

Regard, M, Strauss. E, y Knapp, P (1982). Children's production on verbal and non-verbal fluency tasks. Perceptual and Motor Skills, 55(3) 839-844.

Rey A (1941). L'examen psychologique dans les cas d'encéphalopathie traumatique. Archives de Psychologie, 28, 215-285.

Reynolds, CR (2002). Comprehensive Trail Making Test: Examiner's manual. PRO-ED.

Robertson, IH, Manly, T, Andrade, J, Baddeley, BT, \& Yiend, J (1997). "Oops!": Performance correlates of everyday attentional failures in traumatic brain injured and normal subjects. Neuropsychologia, 35(6), 747-758.

Rumsey, J (1985). Conceptual problem-solving in highly verbal, nonretarded autistic men. Journal of Autism and Developmental Disorders, 15(1), 23-36.

Servera, M y Llabrés, J (2004). CSAT: Tarea de atención sostenida en la infancia. TEA Ediciones.

Shallice, T (1982). Specific impairments of planning. Philosophical transactions of the Royal Society of London. Series B, Biological Sciences, 298(1089), 199-209.

Smith, EE, \& Jonides, J (1999). Storage and executive processes in the frontal lobes. Science, 283(5408), 1657-1661.

* Suárez, F, Mata, B y Peralbo, M (2015). Valoración de un programa de intervención para niños con TEA basado en las TIC. Revista de Estudios e Investigación en Psicología y Educación, (09), 094-098.

*Tachibana, Y, Hwang, Y, Abe, Y, Goto, S, Sugai, K, \& Kawashima, R (2013). Reading aloud improves executive function of children with autism spectrum disorder: a pilot randomized controlled trial. International Journal on Disability and Human Development, 12(1), 91-101.

Thorndike, RL, Hagen, EP, \& Sattler, JM (1986). Stanford-Binet Intelligence Scale (4th ed.). Riverside.
Thorell, LB, \& Nyberg, L (2008). The Childhood Executive Functioning Inventory (CHEXI): A new rating instrument for parents and teachers. Developmental Neuropsychology, 33, $536-552$.

*Tse, CYA, Lee, HP, Chan, KSK, Edgar, VB, Wilkinson-Smith, A, \& Lai, WHE (2019). Examining the impact of physical activity on sleep quality and executive functions in children with autism spectrum disorder: a randomized controlled trial. Autism, 23(7), 1699-1710.

Uzefovsky, F, Allison, C, Smith, P, \& Baron-Cohen, S (2016). Brief report: The Go/No-Go Task online: Inhibitory control deficits in autism in a large sample. Journal of Autism and Developmental Disorders, 46(8), 2774-2779.

Wechsler, D (1981). Manual for the Wechsler Adult Intelligence Scale-Revised. Psychological Corporation.

William, J, Rickert, V, Hogan, J, Zolten, AJ, Satz, P, D'Elia, LF, ... \& Light, R (1995). Children's Color Trails. Archives of Clinical Neuropsychology, 10(3), 211-223.

Wing, L, \& Gould, J (1979). Severe impairments of social interaction and associated abnormalities in children: epidemiology and classification. Journal of autism and developmental disorders, 9(1), 11-29.

Wing, L, \& Potter, D (2002). The epidemiology of autism spectrum disorders: Is the prevalence rising? Mental Retardation and developmental Disabilities Research Reviews, 8(3); 151-161.

(*) artículos incluidos en la revisión 